\title{
Measles outbreaks demand systems-level action locally, nationally and globally
}

\author{
Natasha S. Crowcroft MD(Cantab), Shelly Bolotin PhD
}

Cite as: CMAJ 2019 July 15;191:E777-8. doi: 10.1503/cmaj.190559

CMAJ Podcasts: author interview at https://soundcloud.com/cmajpodcasts/190559-com

$\mathbf{R}$ eports of measles outbreaks are appearing in the news more frequently as we start to face an unfortunate emerging public health problem with no easy solution. The World Health Organization reported a 300\% increase in cases of measles in the first 3 months of 2019, compared with $2018 .{ }^{1}$ There have been outbreaks in countries in all regions of the world, associated with many deaths, including 72 in Europe in $2018 .^{1,2}$ More cases of measles have been reported in the United States so far in 2019 than in any year since the 1990s. ${ }^{3}$ Because it is well known that responding to measles outbreaks is enormously resource intensive and not cost effective compared with prevention, ${ }^{4}$ it is reasonable to ask why these outbreaks are happening and what can be done about them.

Although on the surface the solution - ensuring that all are vaccinated - seems simple, achieving and maintaining universal vaccine coverage is a complex feat. Measles elimination at the local level requires virtually everyone to be vaccinated, which is technically challenging. It has been hard to maintain confidence in immunization. Groups that reject vaccination - whether for philosophical, religious or other reasons - have increased vulnerability to outbreaks in countries that otherwise have strong immunization programs. For example, in Canada and the US, both of which had eliminated measles, importations of the virus from abroad have resulted in an outbreak when it was introduced into communities with low coverage. Because people with similar beliefs can congregate together, such outbreaks will continue to occur in pockets of unimmunized individuals and communities, but will not become widespread as long as the pockets are small enough and overall immunization coverage is high enough. However, vulnerable groups - such as infants, who are too young to be vaccinated, and immunocompromised individuals - will continue to be affected, even in the context of high vaccine coverage in the broader community.

Although those who reject immunization place others at risk of vaccine-preventable diseases, they are not solely to blame. There is a larger systems issue. Sustaining measles elimination requires strong regional public health systems. In a globalized society in which we are all connected, a disease as infectious as measles the most infectious of the vaccine-preventable diseases - is easily

\section{KEY POINTS}

- Recent measles outbreaks have highlighted vaccine hesitancy and the challenges of maintaining confidence in immunization.

- Measles elimination is a technical challenge at the local level because measles is so infectious that it requires virtually everyone to be vaccinated.

- Success relies on having a very high level of political support, excellent data and a very strong immunization system.

- The benefits of using measles vaccination to drive system-level change include increased child health equity.

spread. To prevent this, public health programs need to deliver immunization coverage above the herd immunity threshold which, again, is technically challenging.

Public health programs monitor whether immunization programs are working through vaccine coverage targets that for most vaccine-preventable diseases are set comfortably above the herd immunity threshold, but this is not the case for measles. The threshold is derived from a combination of vaccine effectiveness and the basic reproduction number $R_{0}$ (average number of people infected by each case), which is estimated at 12 to 18 for measles. ${ }^{5}$ Taking the optimistic scenario of 2-dose vaccine effectiveness at $99 \%{ }^{6}$ and $R_{0}=12$, coverage would need to be $92 \%$ to ensure measles doesn't spread. If $R_{0}$ is as high as 18 , or vaccine effectiveness lower than $99 \%$ (which is the more likely scenario), then coverage needs to be greater than $95 \%$. Such coverage is incredibly difficult to achieve. In Canada, only $90 \%$ of 2-year-olds had received at least 1 dose of measles vaccine in $2017 .{ }^{7}$ In the Americas, countries have been asked to aim for $95 \%$ coverage or higher for 2 doses. ${ }^{8}$ During the time that measles elimination was being verified in the Americas, Canada's target was $97 \%$ for at least 1 dose. ${ }^{9}$ Canada has since reduced its target to $95 \%$, but this may not sustain elimination over the long term. ${ }^{10}$

Gaps in immunity will emerge as long as immunization programs are less than perfect. In most countries, however, the quality of information systems, the integration of health care systems and the training of health care providers are not good enough to meet 
the challenge of measles prevention over the long term. In low- and middle-income nations, preventing measles outbreaks is even harder because birth rates are higher, so large pools of susceptible individuals accumulate more rapidly. In these settings, measles vaccine is routinely given at 9 months of age, when its effectiveness is substantially lower. ${ }^{11}$ This necessitates supplementary measles campaigns to fill immunity gaps for older age groups.

Measles outbreaks are indicators of where immunization programs have faltered, but also reveal gaps in child health equity they are the canary in the coal mine that demands immediate change. A bottom-to-top, systems-level approach is needed to ensure that immunization programs reach every child in every country. People and politicians need to know about the dangers of vaccine-preventable illness, and that vaccines are safe and effective. Experience tells us that success in meeting immunization goals relies on having a high level of political support for immunization programs, as well as infrastructure to monitor vaccine coverage with high-quality data.

In the war against microbes, victories are achieved at a huge price, and the peace that follows is fragile. It took many years for the Americas to verify elimination of measles in 2016. It took only 2 years of political disruption in Venezuela to disrupt the health system enough to obliterate this achievement. In some countries, including Canada, there is widespread nonpartisan political support for immunization. However, in many countries this is not the case, owing to war, lack of political will or simply inadequate resources. Smallpox eradication demonstrated the importance of global collaboration. Although we are currently inching forward with a kind of grim determination toward polio eradication, this effort arguably reduces the possibility of a concerted global effort to eradicate measles. Yet it is hard to see how anything other than such an effort would ensure a future that is different from a continuation or deterioration of the current situation.

\section{References}

1. Immunization, vaccines and biologicals: new measles surveillance data for 2019 . Geneva: World Health Organization; 2019. Available: www.who.int/immunization/ newsroom/measles-data-2019/en/ (accessed 2019 May 8).

2. Measles in Europe: record number of both sick and immunized [news release]. Copenhagen (Denmark): World Health Organization (WHO) Regional Office for Europe; 2019 Feb. 7. Available: www.euro.who.int/en/media-centre/sections/press -releases/2019/measles-in-europe-record-number-of-both-sick-and-immunized (accessed 2019 May 8).

3. Measles (rubeola): measles cases and outbreaks. Atlanta: Centers for Disease Control and Prevention; 2019. Available: www.cdc.gov/measles/cases-outbreaks.html (accessed 2019 May 8).

4. Ramsay LC, Crowcroft NS, Thomas S, et al. Cost-effectiveness of measles control during elimination in Ontario, Canada, 2015. Euro Surveill 2019;24:1800370.

5. Guerra FM, Bolotin S, Lim G, et al. The basic reproduction number (R0) of measles: a systematic review. Lancet Infect Dis 2017;17:e420-8.

6. Pillsbury A, Quinn H. An assessment of measles vaccine effectiveness, Australia, 2006-2012. Western Pac Surveill Response J 2015;6:43-50.

7. Preliminary results from the 2017 childhood National Immunization Coverage Survey (cNICS). Ottawa: Public Health Agency of Canada; modified 2019 Apr. 8. Available: www.canada.ca/en/services/health/publications/vaccines-immunization/ vaccine-uptake-canadian-children-preliminary-results-2017-childhood-national -immunization-coverage-survey.html (accessed 2019 May 8).

8. Pan American Health Organization (PAHO); World Health Organization (WHO) Regional Office for the Americas. Proceedings of the 29th Pan American Sanitary Conference, 69th Session of the Regional Committee of WHO for the Americas. Washington (DC): Pan American Health Organization; 2017 Sept. 25-29.

9. Vaccine coverage in Canadian children: results from the 2013 Childhood National Immunization Coverage Survey. Ottawa: Public Health Agency of Canada; 2016.

10. Vaccination coverage goals and vaccine preventable disease reduction targets by 2025. Ottawa: Public Health Agency of Canada; modified 2019 May 8. Available: www.canada.ca/en/public-health/services/immunization-vaccine-priorities/national -immunization-strategy/vaccination-coverage-goals-vaccine-preventable-diseases -reduction-targets-2025.html (accessed 2019 May 8).

11. Lochlainn LN, de Gier B, van der Maas N, et al. Measles vaccination below 9 months of age: systematic literature review and meta-analyses of effects and safety. Bilthoven (The Netherlands): National Institute for Public Health and the Environment; 2015 Sept. 28.

\section{Competing interests: None declared.}

This article was solicited and has not been peer reviewed.

Affiliations: Public Health Ontario (Crowcroft, Bolotin); Laboratory of Medicine and Pathobiology and Dalla Lana School of Public Health, University of Toronto (Crowcroft, Bolotin); ICES (Crowcroft), Toronto, Ont.
Contributors: Both authors contributed to the conception and design of the work, drafted the manuscript, revised it critically for important intellectual content, gave final approval of the version to be published and agreed to be accountable for all aspects of the work.

Acknowledgement: The authors acknowledge Lindsay Friedman's assistance with preparing this article for publication.
Disclaimer: The views and opinions expressed in this article are those of the authors and do not necessarily represent those of, or reflect, the official position of Public Health Ontario.

Correspondence to: Natasha Crowcroft, natasha.crowcroft@oahpp.ca 